\title{
Multi-scale Block Weber Local Descriptor for Face Recognition
}

\author{
Weilan $\mathrm{Pu}^{1, \text { a }}$, XiaoHong Zhang ${ }^{1}$ \\ ${ }^{1}$ College of Software Engineering, Chongqing University, Chongqing 401331, China. \\ apu.weilan@163.com
}

Keywords: Face Recognition, LBP, WLD, MBLBP, FERET

\begin{abstract}
In this paper, we provide a novel local descriptor Multi-scale Block Weber Local Descriptor (MBWLD) for face recognition. It is a LBP-based descriptor which combines MBLBP with WLD for integrating both their advantages. Firstly, Multi-scale block filters is used on the face image to calculate the average gray-values of sub-regions. Secondly, differential excitation and orientation of WLD is extracted using the average neighbors and center. MBWLD extracts both the micro and macro patterns from face image, and becomes more descriptive and robust to face representation. The feasibility and availability are validated by comparing with the standard LBP, MBLBP and WLD on FERET database in expression, illumination and age changes. The experimental result shows that, MBWLD is appropriate for face recognition, and the recognition performance is much well improved especially in illumination and age changes.
\end{abstract}

\section{Introduction}

Face recognition has received crucial attention in recent years due to its widely used in related areas such as identity authentication, access control, surveillance, face retrieval, attendance checking [1]. Methods for face recognition can be generally divided into two categories: global methods and local methods[1]. Recently, local methods have shown promising results in various fields of computer vision. Such as face recognition, texture classification, object detection [2, 3]. Local binary pattern (LBP) [4] is one of the outstanding local methods for image description. As the widely used of LBP, more and more variations based on LBP begin to appear to improve the processing performance, the variations are summarized in [5].

Among the variations, Liao et al. proposed multi-scale block LBP (MBLBP) [6] which replaced the neighbor pixels with the average gray-values of neighbor sub-regions. It encodes not only the micro structures but also macrostructures of image patterns, and enhances the feature description capability. Chen et al. proposed the Weber local descriptor (WLD) [7] inspired by LBP and the Weber's law, it is consist of differential excitation and orientation. Weber's law is based on the fact that human perception of a pattern depends on both the change and the original intensity of a stimulus. Both of the two descriptors are successfully applied to face recognition, and proved to be more descriptive and robust than basic LBP.

However, WLD has the same problem with LBP, it is too local to be robust, and both the micro and macro patterns should be extracted from the face image to enhance the descriptive capability. Thus, in this paper, we propose a new LBP-based descriptor Multi-scale Block WLD (MBWLD) by combining MBLBP with WLD. The descriptor firstly replaces the neighbor pixels with the average gray-values of neighbor sub-regions, and then continue the WLD calculation using the average values. The Sobel descriptor is used to obtain the WLD orientation [8]. Finally, it generated the MBWLD histogram as the image representation. By integrating the advantages of MBLBP and WLD, MBWLD is more descriptive and robust to face representation. 


\section{MBLBP and WLD}

Multi-scale Block LBP. In Multi-scale Block LBP (MB-LBP), the neighbor pixels around a local window in LBP is replaced with average gray-values of neighbor sub-regions. The neighbor subregions are square blocks containing one or more neighbor pixels around the central block. Taking $s \times s$ as the scale of the MB-LBP (the scale is always one of $3 \times 3,9 \times 9,15 \times 15,21 \times 21$. Note that $3 \times 3$ MB-LBP is the original LBP). In MBLBP, average value of image intensity is computed on each neighbor sub-region, and then these values are threshold by the central pixel which is similar to original LBP. Compared to original LBP, MB-LBP captures not only microstructures but also macrostructures of image patterns, and enhances the robustness of the image representation.

Weber Local Descriptor. Weber Local Descriptor (WLD) was inspired by LBP and Weber's Law, which is based on the fact that human could notice a change depends not only on the change of the stimulus but also on the original intensity of the stimulus. For example, one must shout in a loud environment in order to be heard, while whisper is enough in a quiet environment. There are two components of WLD: differential excitation and orientation. The differential excitation is the ratio between the difference values of a central pixel against its neighbors and the gray value of the central pixel. The orientation component is the gradient orientation of the current pixel. The two components are used to construct a concatenated WLD histogram for image description.

\section{Multi-scale Block WLD}

MBWLD. Similar to LBP, original WLD extracts local differential excitation and orientation of an image by comparing the center pixel with its neighbor pixels. While in MBWLD, there are three stages: 1) MB stage: average gray-values of neighbor and center sub-regions are firstly computed as the neighbor values and center value, the scale is one of $3 \times 3,9 \times 9,15 \times 15,21 \times 21$; $)$ WLD stage: differential excitation and orientation of the current pixel are extracted using the values generated in stage one, and the MBWLD histogram is generated the same with WLD. The two stages of MBWLD is shown in Figure 1.

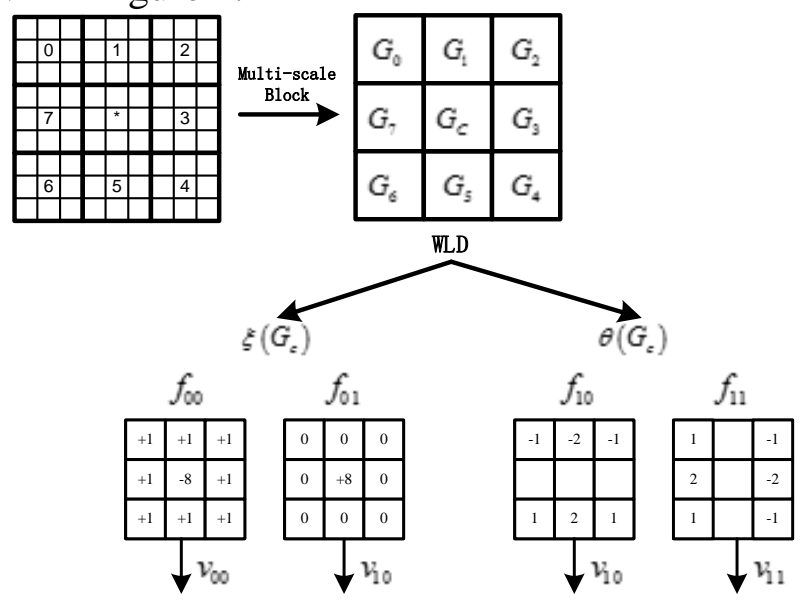

Figure 1. MB and WLD stage of MBWLD

In the formulas of MBWLD, the main modification is replacing the pixel value with sub-region average value. The stimulus change and stimulus of differential excitation and orientation is defined as following:

$$
\begin{aligned}
& v_{s}^{00}=\sum_{i=0}^{p-1}\left(\Delta G_{i}\right)=\sum_{i=0}^{p-1}\left(G_{i}-G_{c}\right), v_{s}^{01}=G_{c} \\
& v_{s}^{10}=\left(G_{6}+2 G_{5}+G_{4}\right)-\left(G_{0}+2 G_{1}+G_{2}\right), v_{s}^{11}=\left(G_{0}+2 G_{7}+G_{6}\right)-\left(G_{2}+2 G_{3}+G_{4}\right)
\end{aligned}
$$

where $G$ is the average gray-value of sub-region. We use the Sobel descriptor as the orientation component of MBWLD to obtain more detailed gradient information. The orientation filters $f_{10}$ and $f_{11}$ is shown in Figure 1. 
Differential Excitations Histogram. In [7], they plot an average histogram of the differential excitations on 2,000 texture images. The histogram shows that in $[-\pi / 2, \pi / 2]$, there are more frequencies at the two sides (high frequency) of the average histogram. In this paper, we plot an average histogram of the differential excitations of both original WLD and MBWLD on 2,000 face images. Figure 2 (a) shows the average histogram of WLD differential excitations. Figure 2 (b) shows the average histogram of MBWLD differential excitations. The average histogram is more equalized, and very similar to that of the WLD on texture images. Thus, after MB step, the differential excitations is proved to be more descriptive, and the weights for WLD histogram is more appropriate for it.

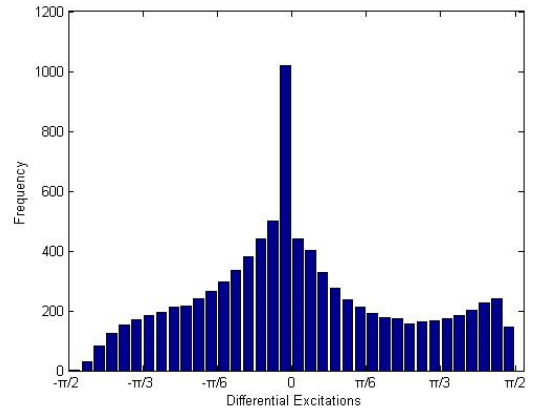

(a)

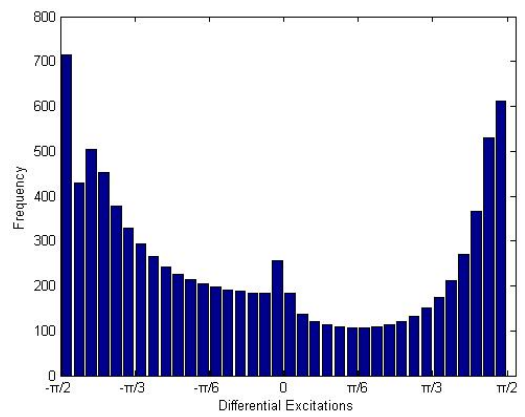

(b)

Figure 2. Average histogram of the differential excitation on 2,000 face images: (a) original WLD, (b) MBWLD

\section{Experimental Result and Analysis}

Datasets. FERET database contains 14051 gray-scale images for 1199 persons with different conditions in expression, illumination, shooting time, pose angle, etc. [9] . In the usual FERET evaluation protocol, there are five subsets for evaluation: fa (1195 images for 1195 persons), fb (1195 images for 1195 persons), fc (194 images for 194 persons), dup I (722 images for 243 persons), dup II (234 images for 75 persons). In FERET, fa is used as the gallery, and the four others are probes: $\mathrm{fb}$ is the expression probe, fc is the illumination probe, and dup I and dup II are both the age probe, where dup II is a more difficult subset of dup I.

Performance Comparison with Existing Descriptors. The CMC curves of descriptors in condition changes on FERET are shown in Figure 3. CMC plots the recognition rate as a function of rank $n$, the recognition rate increases to $100 \%$ with the rank growth.

In expression changes, all the tested descriptors are robust to expression changes, they get the recognition rate more than $95 \%$ when rank is 1 . MBLBP has a slightly better recognition rate at beginning, and the CMC curves are very close to each other after rank is 3 . In illumination changes, MBWLD gets the best recognition rate from the beginning, WLD and LBP are next only to MB. The recognition rate of MBLBP is only 20\% when rank is 1, it is seriously influenced by illumination. However, by combining with WLD, the performance improved prominently. In age changes, on dup I, the recognition rate of MBWLD, MBLBP and LBP is similar from the beginning, and that of WLD is a little less than others. On dup II, MBWLD has the best recognition rate again, and exceeds all others a little all the time. MBLBP and WLD have almost the same CMC curve on dup II, and their combination successfully improve the performance again. 


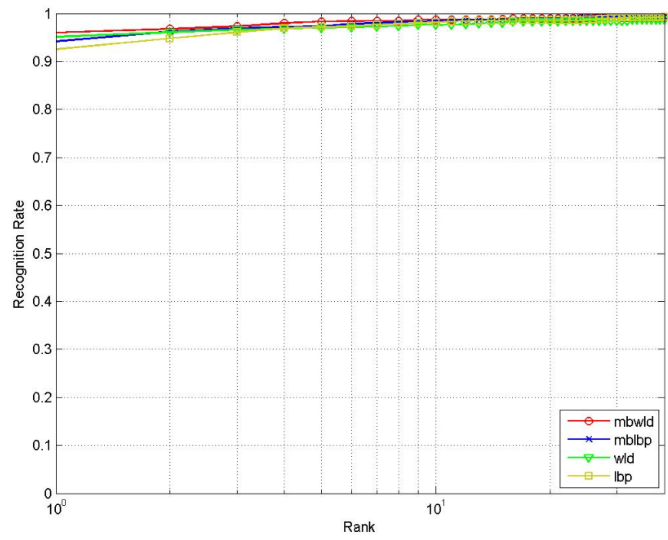

(a)

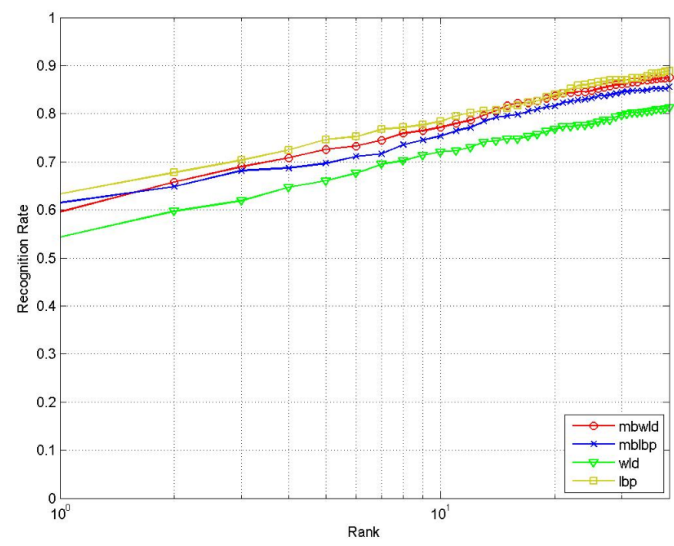

(c)

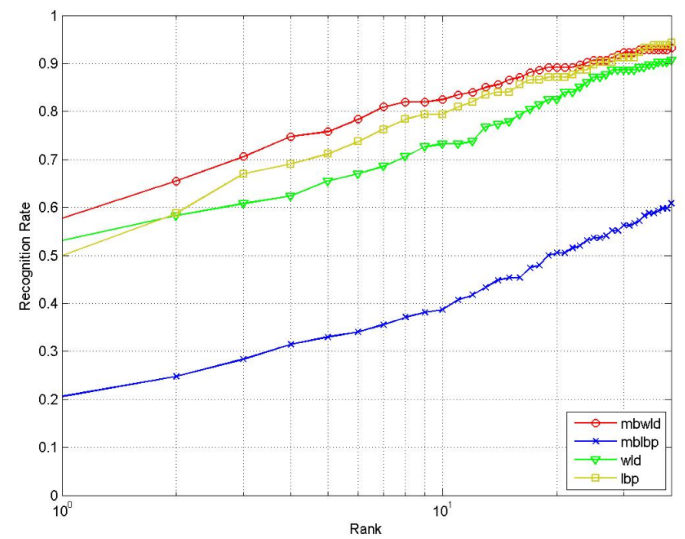

(b)

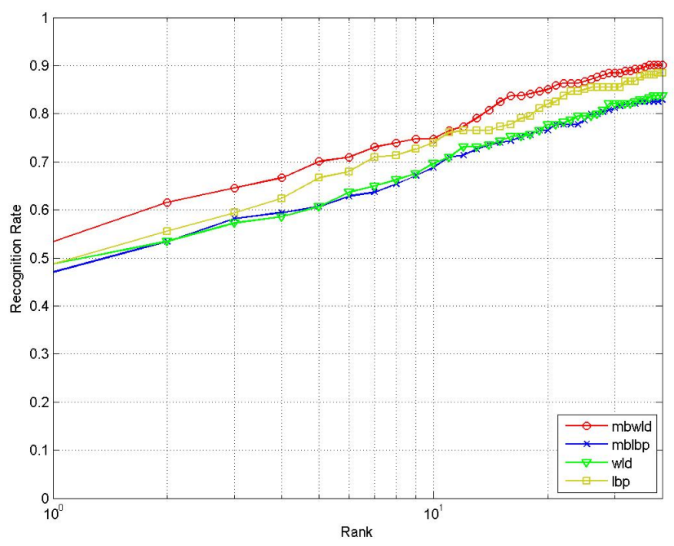

(d)

Figure 3. The CMC curve of descriptors in condition changes: (a) expression, (b) illumination, (c) age I, (d) age II

\section{Conclusion}

In this paper, we present a Multi-scale Block Weber Local Descriptor (MBWLD) by combining MBLBP with WLD. It firstly calculates the average gray-values of neighbor sub-regions, and then extracts the differential excitation and orientation of WLD by using the average values. By integrating the advantages of MBLBP and WLD, the differential excitation statistic histogram is more equalized. This makes MBWLD become more descriptive and robust to face representation.

Experiments are taking on FERET database in condition changes. The experimental results show that MBWLD is appropriate for face recognition in condition changes, and the performance of MBLBP is much well improved especially in illumination changes and age changes. In our further work, we will consider to improve the computing efficiency and performance by extracting other new local features, but not just combine the existing descriptors.

\section{References}

[1] W. Zhao, R. Chellappa, Face recognition: A literature survey, Acm Comput. Surv. 35 (2003) 399-458.

[2] R. Mehta, J. Yuan, K. Egiazarian, Face recognition using scale-adaptive directional and textural features, Pattern Recognit. 47 (2014) 1846-1858.

[3] W. Xia, S. Yin, P. Ouyang, A high precision feature based on LBP and Gabor theory for face recognition., Sensors (Basel). 13 (2013) 4499-4513. 
[4] T. Ahonen, A. Hadid, M. Pietikäinen, Face description with local binary patterns: application to face recognition, IEEE Trans. Pattern Anal. Mach. Intell. 28 (2006) 2037-41.

[5] M. Bereta, W. Pedrycz, M. Reformat, Local descriptors and similarity measures for frontal face recognition: A comparative analysis, J. Vis. Commun. Image Represent. 24 (2013) 1213-1231.

[6] S. Liao, X. Zhu, Z. Lei, L. Zhang, S. Li, Learning multi-scale block local binary patterns for face recognition, Adv. Biometrics. (2007) 828-837.

[7] J. Chen, S. Shan, C. He, G. Zhao, M. Pietikäinen, X. Chen, et al., WLD: a robust local image descriptor, IEEE Trans. Pattern Anal. Mach. Intell. 32 (2010) 1705-1720.

[8] D. Gong, S. Li, Y. Xiang, Face recognition using the Weber Local Descriptor, in: 1st Asian Conf. Pattern Recognition, ACPR 2011: pp. 589-592.

[9] P.J. Phillips, H. Wechsler, J. Huang, P.J. Rauss, The FERET database and evaluation procedure for face-recognition algorithms, Image Vis. Comput. 16 (1998) 295-306. 